\title{
La QUALAB ou l'importance des contrôles
}

Service tarifaire de la FMH

La Commission suisse pour l'assurance qualité dans le laboratoire médical (QUALAB) existe depuis de nombreuses années. Elle a pour attribution d'ordonner des mesures de contrôle de la qualité dans les laboratoires de cabinets médicaux, ce en vertu de la Loi sur l'assurance-maladie (LAMal), de l'Ordonnance sur l'assurancemaladie (OAMAL) et de la liste des analyses publiée par le Département fédéral de l'intérieur (DFI).

Vous trouverez de nombreuses informations à ce sujet sur le site internet de la QUALAB (www.qualab.ch). Vous pourrez notamment y consulter la liste actualisée des analyses soumises au contrôle de qualité externe. La version en vigueur pour 2005 est reproduite à la fin du présent article.

\section{Contrôle de qualité obligatoire}

Quelque 8000 membres de la FMH ont un laboratoire d'analyses médicales dans leur cabinet. Chaque praticien qui facture, à la charge des assurances, des analyses soumises au contrôle de qualité, doit obligatoirement participer aux contrôles externes prescrits et s'affilier à l'un des trois centres de contrôle de qualité reconnus:

- CSCQ Centre Suisse de Contrôle de Qualité, Chemin du Petit-Bel-Air 2, 1225 ChêneBourg, tél. 0223055230 (français), tél. 022 3055231 (allemand), tél. 0223055232 (italien), e-mail: cscq@hcuge.ch, site internet: www.cscq.ch;

- MQ Verein für medizinische Qualitätskontrolle, c/o Institut de chimie clinique, Hôpital universitaire, 8091 Zurich, tél. 04425534 11, e-mail: mq@unizh.ch, site internet: www. mqzh.ch;

- STS CRS Service de transfusion sanguine de la CRS bernoise SA, Murtenstrasse 133, case postale 5512, 3001 Berne, tél. 0313842323.
Correspondance:

Service tarifaire de la FMH

Gösgerstrasse 8

CH-4600 Olten

E-mail: fmhtarif@hin.ch

\section{Sanctions en cas de non-participation aux contrôles de qualité externes}

Si l'on constate durant deux années consécutives qu'un médecin ne s'est pas soumis aux contrôles de qualité externes obligatoires, celui-ci est inscrit dans une liste négative. Une telle liste a été introduite pour la première fois à la fin 2004 et comptait alors environ 150 personnes - soit moins de $2 \%$ des médecins facturant des analyses - dont les noms ont été communiqués aux assureurs conformément aux réglementations en vigueur. Dès 2005, les assureurs ont le droit de refuser pendant une année le remboursement des prestations de laboratoire facturées par les médecins figurant sur cette liste.

Tout médecin qui figure sur cette liste et souhaite s'opposer à cette décision peut s'adresser à la Commission paritaire de confiance de la QUALAB (Mme D. Braun, santésuisse, Römerstrasse 20, 4502 Soleure, tél. 03262542 23, fax 03262542 72). Lorsqu'un recours est interjeté auprès de cette commission, cette dernière doit siéger dans les 6 mois et le recourant obtient l'effet suspensif, autrement dit, il peut continuer à facturer ses analyses à la charge des assurances sociales.

A l'avenir, de telles listes, tant négatives que positives, seront établies une fois l'an. Par conséquent, il est impératif que chaque médecin qui facture des analyses médicales à la charge des assurances sociales prenne connaissance des communiqués de la QUALAB.

\section{Listes des analyses soumises dès le $\mathbf{1}^{\text {er }}$ janvier 2005 au contrôle de qualité externe obligatoire}

\section{Chimie, hématologie, immunologie}

Les paramètres ci-dessus sont aussi soumis au contrôle de qualité externe obligatoire, lorsqu'ils sont facturés dans le cadre d'un bloc d'analyses sous un autre numéro ou si le résultat est obtenu suite à un test clinique. 


\begin{tabular}{|c|c|c|c|c|c|}
\hline \multirow[t]{4}{*}{ M } & no position & paramètre & $\begin{array}{l}\text { critères } \\
\text { de qualité }\end{array}$ & $\begin{array}{l}\text { critères } \\
\text { de nombre }\end{array}$ & CCQ reconnus \\
\hline & 8000.00 & Groupes sanguins & juste & $11 / 12$ & C \\
\hline & 8006.00 & Alanine-aminotransférase (ALAT) & $\pm 25 \%$ & $75 \%$ & $a, b$ \\
\hline & 8007.00 & Albumine, chimique & $\pm 15 \%$ & $75 \%$ & $a, b$ \\
\hline \multirow[t]{8}{*}{$N$} & 8017.00 & Alpha-1-foetoprotéine (AFP) & $\pm 25 \%$ & $75 \%$ & $a, b$ \\
\hline & 8012.00 & Phosphatase alcaline & $\pm 25 \%$ & $75 \%$ & $a, b$ \\
\hline & 8036.00 & Amphétamines, ql & juste & $75 \%$ & $a, b$ \\
\hline & 8037.00 & Amylase & $\pm 40 \%$ & $75 \%$ & $a, b$ \\
\hline & 8058.00 & Aspartate-aminotransférase (ASAT) & $\pm 25 \%$ & $75 \%$ & $a, b$ \\
\hline & 8116.00 & Barbituriques, ql & juste & $75 \%$ & $a, b$ \\
\hline & 8119.00 & Benzodiazépines, ql & juste & $75 \%$ & $a, b$ \\
\hline & 8126.00 & Bilirubine totale & $\pm 20 \%$ & $75 \%$ & $a, b$ \\
\hline C & 8129.00 & Gazométrie: $\mathrm{pH}$ & $\pm 1 \%$ & $75 \%$ & $a, b$ \\
\hline \multirow[t]{3}{*}{ C } & 8129.00 & Gazumétrie: $\mathrm{pO}_{2}, \mathrm{pCO}_{2}$ & $\pm 15 \%$ & $75 \%$ & $a, b$ \\
\hline & 8137.00 & Protéine $\mathrm{C}$ réactive (CRP), qn & $\pm 20 \%$ & $75 \%$ & $a, b$ \\
\hline & 8149.00 & Calcium total & $\pm 12 \%$ & $75 \%$ & $a, b$ \\
\hline \multirow[t]{4}{*}{$\mathrm{N}$} & 8152.00 & Antigène carcino-embryonnaire (CEA) & $\pm 25 \%$ & $75 \%$ & $a, b$ \\
\hline & 8156.00 & Chlorures & $\pm 10 \%$ & $75 \%$ & $a, b$ \\
\hline & 8158.00 & Cholestérol total & $\pm 10 \%$ & $75 \%$ & $a, b$ \\
\hline & 8169.00 & Cocaïne, ql & juste & $75 \%$ & $a, b$ \\
\hline \multirow[t]{17}{*}{$\mathrm{N}$} & 8174.00 & Cortisol, basal & $\pm 20 \%$ & $75 \%$ & $a, b$ \\
\hline & 8179.00 & D-dimère, ql & juste & $75 \%$ & $a, b$ \\
\hline & 8179.01 & D-dimère, qn & $\pm 30 \%$ & $75 \%$ & $a, b$ \\
\hline & 8184.00 & Cannabis, ql & juste & $75 \%$ & $a, b$ \\
\hline & 8192.00 & Fer & $\pm 20 \%$ & $75 \%$ & $a, b$ \\
\hline & 8199.00 & Erythrocytes alloanticorps, spécification & juste & $100 \%$ & C \\
\hline & 8200.00 & $\begin{array}{l}\text { Erythrocytes alloanticorps, test de } \\
\text { recherche }\end{array}$ & juste & $100 \%$ & C \\
\hline & 8210.00 & Erythrocytes, numération & $\pm 25 \%$ & $75 \%$ & $a, b$ \\
\hline & 8225.00 & Ferritine & $\pm 25 \%$ & $75 \%$ & $a, b$ \\
\hline & 8229.00 & Fibrinogène, d'après Clauss & $\pm 15 \%$ & $75 \%$ & $a, b$ \\
\hline & 8239.00 & Folates & $\pm 20 \%$ & $75 \%$ & $a, b$ \\
\hline & 8249.00 & Gamma-glutamyl-transpeptidase ( $\gamma$-GT) & $\pm 25 \%$ & $75 \%$ & $a, b$ \\
\hline & 8259.00 & Glucose & $\pm 10 \%$ & $75 \%$ & $a, b$ \\
\hline & 8265.00 & Hémoglobine glyquée (HbA1c) & $\pm 15 \%$ & $75 \%$ & $a, b$ \\
\hline & 8273.00 & Hématocrite & $\pm 12 \%$ & $75 \%$ & $a, b$ \\
\hline & 8275.00 & Hémoglobine & $\pm 12 \%$ & $75 \%$ & $a, b$ \\
\hline & 8284.00 & Urée & $\pm 20 \%$ & $75 \%$ & $a, b$ \\
\hline \multirow[t]{12}{*}{$\mathrm{N}$} & 8286.00 & Human Choriongonadotropin (HCG), qn & $\pm 25 \%$ & $75 \%$ & $a, b$ \\
\hline & 8288.00 & $\begin{array}{l}\text { Cholestérol HDL sans précipitation } \\
\text { séparée }\end{array}$ & $\pm 30 \%$ & $75 \%$ & $a, b$ \\
\hline & 8313.00 & Immunoglobuline IgA (sérum) & $\pm 25 \%$ & $75 \%$ & $a, b$ \\
\hline & 8317.00 & Immunoglobuline IgE total, qn & $\pm 20 \%$ & $75 \%$ & $a, b$ \\
\hline & 8317.01 & Immunoglobuline lgE multispécifique & juste & $75 \%$ & $a, b$ \\
\hline & 8317.02 & IgE spécifique - arachides & \pm 1 classe & $75 \%$ & $a, b$ \\
\hline & 8317.02 & IgE spécifique - bouleau & \pm 1 classe & $75 \%$ & $a, b$ \\
\hline & 8317.02 & IgE spécifique - épithélium du chat & \pm 1 classe & $75 \%$ & $a, b$ \\
\hline & 8317.03 & IgE spécifique - arachides qn & $\pm 20 \%$ & $75 \%$ & $a, b$ \\
\hline & 8317.03 & IgE spécifique - bouleau qn & $\pm 20 \%$ & $75 \%$ & $a, b$ \\
\hline & 8317.03 & IgE spécifique - épithélium du chat qn & $\pm 20 \%$ & $75 \%$ & $a, b$ \\
\hline & 8318.00 & Immunoglobuline IgG (sérum) & $\pm 25 \%$ & $75 \%$ & $a, b$ \\
\hline
\end{tabular}




\begin{tabular}{|c|c|c|c|c|c|}
\hline M & no position & paramètre & $\begin{array}{l}\text { critères } \\
\text { de qualité }\end{array}$ & $\begin{array}{l}\text { critères } \\
\text { de nombre }\end{array}$ & $\mathrm{CCQ}$ reconnus \\
\hline & 8321.00 & Immunoglobuline IgM (sérum) & $\pm 25 \%$ & $75 \%$ & $a, b$ \\
\hline & 8343.00 & Potassium & $\pm 10 \%$ & $75 \%$ & $a, b$ \\
\hline & 8384.00 & Créatine-kinase (CK), total & $\pm 40 \%$ & $75 \%$ & $a, b$ \\
\hline & 8387.00 & Créatinine & $\pm 20 \%$ & $75 \%$ & $a, b$ \\
\hline & 8394.00 & Lactate-déshydrogénase (LDH) & $\pm 25 \%$ & $75 \%$ & $a, b$ \\
\hline & 8403.00 & Leucocytes, répartition & * & $75 \%$ & $a, b$ \\
\hline & 8406.00 & Leucocytes, numération & $\pm 25 \%$ & $75 \%$ & $a, b$ \\
\hline & 8428.00 & Magnésium & $\pm 20 \%$ & $75 \%$ & $a, b$ \\
\hline & 8433.00 & Méthadone, ql & juste & $75 \%$ & $a, b$ \\
\hline & 8437.00 & Myoglobine & $\pm 30 \%$ & $75 \%$ & $a, b$ \\
\hline & 8438.00 & Sodium & $\pm 10 \%$ & $75 \%$ & $a, b$ \\
\hline & 8444.00 & Opiacés, ql & juste & $75 \%$ & $a, b$ \\
\hline & 8450.00 & Amylase pancréatique & $\pm 25 \%$ & $75 \%$ & $a, b$ \\
\hline & 8455.10 & $\begin{array}{l}\text { Temps de thromboplastine partielle } \\
\text { activée (aPTT) }\end{array}$ & $\pm 25 \%$ & $75 \%$ & $a, b$ \\
\hline & 8462.00 & Phosphate & $\pm 15 \%$ & $75 \%$ & $a, b$ \\
\hline & 8485.00 & Prostate, antigène spécifique (PSA) & $\pm 25 \%$ & $75 \%$ & $a, b$ \\
\hline & 8485.01 & Prostate, antigène spécifique (PSA), libre & $\pm 25 \%$ & $75 \%$ & $a, b$ \\
\hline & 8492.00 & Protéines totales & $\pm 15 \%$ & $75 \%$ & $a, b$ \\
\hline & 8548.00 & Quick & $\pm 15 \%$ & $75 \%$ & $a, b$ \\
\hline & 8560.00 & Thrombocytes, numération & $\pm 25 \%$ & $75 \%$ & $a, b$ \\
\hline & 8562.00 & Thyroxine libre (FT4) & $\pm 20 \%$ & $75 \%$ & $a, b$ \\
\hline & 8572.00 & Triglycérides & $\pm 20 \%$ & $75 \%$ & $a, b$ \\
\hline & 8574.10 & Troponine (T ou I), par méthode ELISA & $\pm 30 \%$ & $75 \%$ & $a, b$ \\
\hline & 8576.00 & Thyréotropine (TSH), basale & $\pm 20 \%$ & $75 \%$ & $a, b$ \\
\hline & 8577.00 & Thyréotropine (TSH), stimulée & $\pm 20 \%$ & $75 \%$ & $a, b$ \\
\hline & 8578.00 & Urate & $\pm 20 \%$ & $75 \%$ & $a, b$ \\
\hline & 8587.00 & $\begin{array}{l}\text { Test de compatibilité, test ind. antiglob. } \\
\text { humaines }\end{array}$ & juste & $100 \%$ & C \\
\hline & 8590.00 & Vitamine B12 & $\pm 20 \%$ & $75 \%$ & $a, b$ \\
\hline \multicolumn{6}{|c|}{$\begin{array}{l}M=\text { modifications par rapport à 2004: } N=\text { nouveau; } C=\text { changement de critère; } S=\text { supprimé; } Q=\text { changement } \\
\text { de CCQ reconnu. }\end{array}$} \\
\hline
\end{tabular}

\section{Microbiologie}

Virologie

\begin{tabular}{|c|c|c|c|c|}
\hline 9108.40 & $\begin{array}{l}\text { Hépatite } B \text {, virus, } H B s \text {, recherche } \\
\text { des antigènes, ql }\end{array}$ & pas de faux nég. & $100 \%$ & $a, c$ \\
\hline 9109.01 & Hépatite $C$, virus, anticorps $\lg$ ou lgG, ql & pas de faux nég. & $100 \%$ & $a, c$ \\
\hline 9116.01 & $\begin{array}{l}\text { HIV } 1+2 \text {, dépistage des anticorps } \\
(\text { EIA), ql }\end{array}$ & pas de faux nég. & $100 \%$ & $a, c$ \\
\hline 9116.40 & $\begin{array}{l}\text { HIV } 1+2 \text {, dépistage des anticorps } \\
\text { (test rapide), ql }\end{array}$ & pas de faux nég. & $100 \%$ & $a, b$ \\
\hline
\end{tabular}




\section{Bactériologie}

\begin{tabular}{|c|c|c|c|c|c|}
\hline $\mathrm{N}$ & & $\begin{array}{l}\text { L'ensemble des analyses de bactériologie } \\
\text { du chapitre } 3.2 .2 \text { de la liste fédérale } \\
\text { des analyses }\end{array}$ & & $75 \%$ & $a, b$ \\
\hline \multicolumn{6}{|c|}{ Analyses des soins de base (chapitre 5.1.3 de la liste des analyses) } \\
\hline & 9309.00 & Urine slide & juste & $75 \%$ & $a, b$ \\
\hline $\mathrm{N}$ & 9559.72 & $\begin{array}{l}\text { Streptococcus bêta-hémolytique du } \\
\text { groupe A (par méthode rapide) }\end{array}$ & juste & $75 \%$ & $a, b$ \\
\hline
\end{tabular}

\section{Remarque}

Le contrôle de qualité effectif pour les analyses du chapitre 3.2.2 LA est réalisé sur mandat du CSCQ et de MQ par les centres de contrôle suivants: NEQAS (pour le CSCQ) et IMM (pour MQ). CSCQ et MQ assurent le contact avec les laboratoires. Une adhésion directe à NEQAS ou IMM n'est pas reconnue dans le cadre du contrôle de qualité obligatoire.

\section{Génétique}

Pour des raisons techniques, un seul contrôle externe est organisé pour ces analyses. Par contre, plusieurs échantillons doivent être examinés lors de ce contrôle.

Tous les laboratoires qui effectuent les analyses ci-dessous doivent envoyer chaque année à la QUALAB une copie de l'évaluation resp. du certificat, et ceci dès réception du document, mais au plus tard pour la fin de l'année.

\begin{tabular}{|c|c|c|c|c|c|}
\hline M & no position & paramètre/maladie & $\begin{array}{l}\text { critères } \\
\text { de qualité }\end{array}$ & $\begin{array}{l}\text { critères } \\
\text { de nombre }\end{array}$ & CCQ reconnus \\
\hline & 8801.00 & Caryotype constitutionnel & juste & $1 / 1$ & $e, f$ \\
\hline & 8802.00 & $\begin{array}{l}\text { Analyse chromosomique, hémopathies } \\
\text { malignes }\end{array}$ & juste & $1 / 1$ & $\mathrm{~F}$ \\
\hline & 8805.00 & FISH métaphase & juste & $1 / 1$ & $e, f$ \\
\hline & 8806.00 & FISH interphase & juste & $1 / 1$ & $e, f$ \\
\hline & 8810.01 & Angelman / Prader Willi & juste & $1 / 1$ & $d, e$ \\
\hline & 8810.05 & Microdélétion du chromosome $Y$ & juste & $1 / 1$ & $\mathrm{D}$ \\
\hline & 8810.07 & Mucoviscidose & juste & $1 / 1$ & d, e \\
\hline & 8810.08 & Syndrome de I'X-fragile & juste & $1 / 1$ & d, e \\
\hline & 8810.11 & Hémochromatose & juste & $1 / 1$ & $a, b, e$ \\
\hline & 8810.13 & Maladies d'hémostase & juste & $1 / 1$ & $a, b$ \\
\hline & 8810.19 & $\begin{array}{l}\text { Cancer du sein / Carcinomes du colon } \\
\text { héréditaires / Rétinoblastome }\end{array}$ & juste & $1 / 1$ & $d, e$ \\
\hline & 8810.23 & Dystrophie musculaire de Duchenne & juste & $1 / 1$ & $d, e$ \\
\hline & 8810.27 & $\begin{array}{l}\text { Dystrophie myotonique / Ataxie } \\
\text { de Friedreich / Huntington Disease / } \\
\text { Ataxies spinocérébelleuse }\end{array}$ & juste & $1 / 1$ & $\mathrm{E}$ \\
\hline & 8810.32 & Charcot-Marie-Tooth & juste & $1 / 1$ & $d, e$ \\
\hline
\end{tabular}

